\title{
Promise of doublet chemotherapy
}

The survival duration of patients with pancreatic adenocarcinoma (PDAC) is among the lowest of all cancers. For patients who present with resectable PDAC, adjuvant gemcitabine-based chemotherapy is the standard treatment; however, survival improvements have been noted in patients receiving doublet gemcitabline and platinumbased therapy. In light of the promise of the combination of gemcitabine and cisplatin, a phase II trial was conducted to prospectively evaluate the outcomes of patients treated with this combination regimen following resection of PDAC.

The trial did not meet the full planned accrual, with only 22 of the target 45 patients enrolled. The primary end points were recurrence-free survival (RFS) and overall survival. Despite the small cohort size, at a median follow-up duration of 37.5 months, RFS was 16.7 months and overall survival was 35.5 months. These outcomes compare favourably with historical data, and indicate an improvement on the current standard of care. The toxicity profile was also acceptable, illustrating that combining two agents did not increase toxicity substantially.

Low expression of the excision repair protein ERCC1 has been implicated as a predictor of a favourable response to platinum therapy. In this small study, immunohistochemically confirmed ERCC1 expression was not associated with any significant relationship with RFS or overall survival, with limited predictive biomarker value; however, there was a trend towards superior outcomes in patients with low levels of ERCC1 expression.

In summary, patients who received the cisplatin and gemcitabline doublet had good survival outcomes compared with other historical studies. ERCC1 expression was easily evaluated, and its role as a putative predictive biomarker should be studied in a larger prospective trial.

\section{Lisa Hutchinson}

ORIGINAL ARTICLE Postlewait, L. M. et al. Combination gemcitabine/cisplatin therapy and ERCC1 expression for resected pancreatic adenocarcinoma: results of a phase II prospective trial.J.Surg. Oncol. http://dx.doi.org/10.1002/ jso.24317 (2016) 